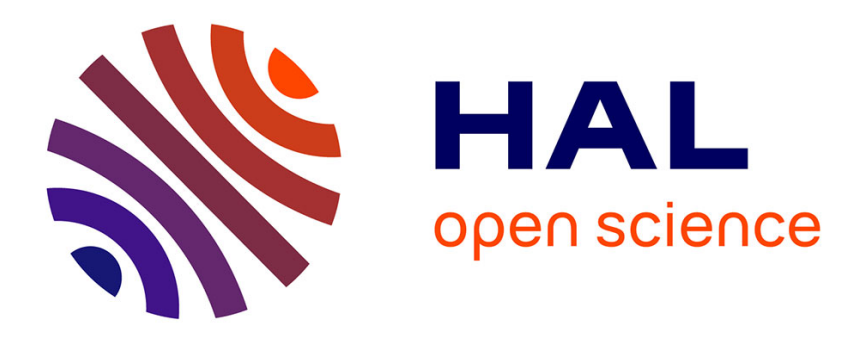

\title{
Cristal trapézoïdal en spectroscopie X d'implosion par laser
}

\author{
A. Poquerusse, J.-M. Boudenne
}

\section{To cite this version:}

A. Poquerusse, J.-M. Boudenne. Cristal trapézoïdal en spectroscopie X d'implosion par laser. Revue de Physique Appliquée, 1988, 23 (3), pp.315-318. 10.1051/rphysap:01988002303031500 . jpa-00245775

\section{HAL Id: jpa-00245775 https://hal.science/jpa-00245775}

Submitted on 1 Jan 1988

HAL is a multi-disciplinary open access archive for the deposit and dissemination of scientific research documents, whether they are published or not. The documents may come from teaching and research institutions in France or abroad, or from public or private research centers.
L'archive ouverte pluridisciplinaire $\mathbf{H A L}$, est destinée au dépôt et à la diffusion de documents scientifiques de niveau recherche, publiés ou non, émanant des établissements d'enseignement et de recherche français ou étrangers, des laboratoires publics ou privés. 


\title{
Cristal trapézoïdal en spectroscopie $\mathbf{X}$ d'implosion par laser
}

\author{
A. Poquerusse $\left({ }^{1,2}\right)$ et J.-M. Boudenne $\left({ }^{1}\right)$ \\ (1) GRECO Interaction Laser-Matière, Ecole Polytechnique, 91128 Palaiseau Cedex, France \\ ( $\left.{ }^{2}\right)$ Laboratoire de Physique des Milieux Ionisés, Ecole Polytechnique, 91128 Palaiseau Cedex, France
}

(Reçu le 2 octobre 1987, accepté le 16 décembre 1987)

\begin{abstract}
Résumé. - En courbant élastiquement un cristal trapézoïdal pour la diffraction de Bragg des rayons $\mathrm{X}$, on réalise un spectrographe à champ plan, large bande et haute résolution, sous incidence normale. Grâce à lui, on étudie des implosions de microballons par un laser à six faisceaux.
\end{abstract}

Abstract. - Elastically bent, trapezoidal crystals for Bragg diffraction of X-rays allow designing broadband spectrometers with flat field, normal incidence and high resolution. This is applied to study microballoon implosions driven by six laser beams.

\section{Introduction.}

Un article précédent [1] montrait l'intérêt d'employer un cristal mince doté par flexion élastique d'un gradient de concavité, pour réaliser un spectrographe à champ plan, sous incidence quasi normale, et atteignant sur une grande partie du spectre la résolution ultime permise par la diffraction de Bragg des rayons $\mathrm{X}$ sur ce cristal. Toutefois, en se contentant banalement d'une forme rectangulaire, on restreignait le champ des possibilités. De fait, nous montrons ici qu'une simple forme trapézoïdale étend suffisamment la latitude de choix pour maximiser le pouvoir de résolution sur tout le spectre.

Remarquons, pour l'anecdote, que la forme trapézoïdale est intermédiaire entre la forme rectangulaire et la forme triangulaire, qui est couramment employée [2] sur les sources de rayonnement synchrotron, pour réaliser facilement une courbure uniforme réglable.

L'application plus particulièrement présentée ici portera sur une expérience d'implosion de microballons par les six faisceaux ultra-violets du laser du GRECO ILM. La figure 1 en donne l'aspect général.

\section{Réflexion de Bragg sur un cristal arbitrairement} courbé.

Nous n'envisageons que le cas de la réflexion de Bragg symétrique, c'est-à-dire sur des plans réticulaires (distants de $d$ ) parallèles à la surface du cristal. La longueur d'onde $\lambda$, l'angle d'attaque $\theta$ et l'ordre

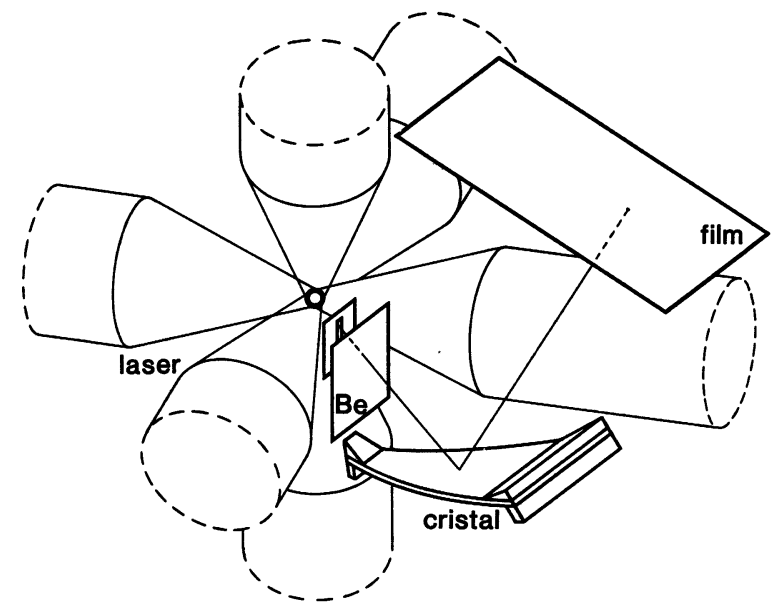

Fig. 1. - Schéma du dispositif expérimental.

[Outline of the experimental setup.]

de diffraction $n$ sont alors soumis à la relation de Bragg bien connue :

$$
n \lambda=2 d \sin \theta .
$$

La concavité imposée au cristal a pour vertu essentielle d'autoriser une réduction considérable du grandissement longitudinal $g$, rapport de la largeur géométrique de la raie spectrale sur le détecteur au diamètre $D$ d'une source monochromatique, le profil angulaire de la diffraction de Bragg étant supposé 
infiniment étroit. L'expression de $g$, dont [1] esquisse la démonstration ainsi que celle de la formule suivante, introduit le trajet $L_{1}$ de la source au cristal, où le rayon de courbure local est $R$ (dans le plan d'incidence), puis le trajet $L_{2}$ du cristal au détecteur, atteint sous l'angle d'incidence $\beta$ :

$$
g=\frac{1}{\cos \beta} \frac{R \sin \theta-L_{2}}{R \sin \theta-L_{1}}
$$

La qualité d'une configuration particulière sourcecristal-détecteur se jugera en comparant $g D$ à toute autre cause d'élargissement des raies, soit propre au détecteur (grain photographique, fente de microdensitomètre, pas d'une mosaïque semi-conductrice, ...), soit propre au milieu-source (effet recherché), soit propre au cristal (effet inévitable), sachant qu'une largeur totale à mi-hauteur $\Delta \lambda$ se traduit par une largeur mesurée sur le détecteur :

$$
\Delta x=\frac{\Delta \lambda}{\lambda} \tan \theta\left(\frac{L_{2}}{\cos \beta}+g L_{1}\right) .
$$

\section{Cristal trapézoïdal élastiquement courbé.}

Si un cristal de forme allongée et d'épaisseur constante est maintenu en flexion élastique par des appuis proches des extrémités, le moment fléchissant varie linéairement dans l'intervalle libre et est proportionnel à la largeur locale $\ell$ et à la courbure

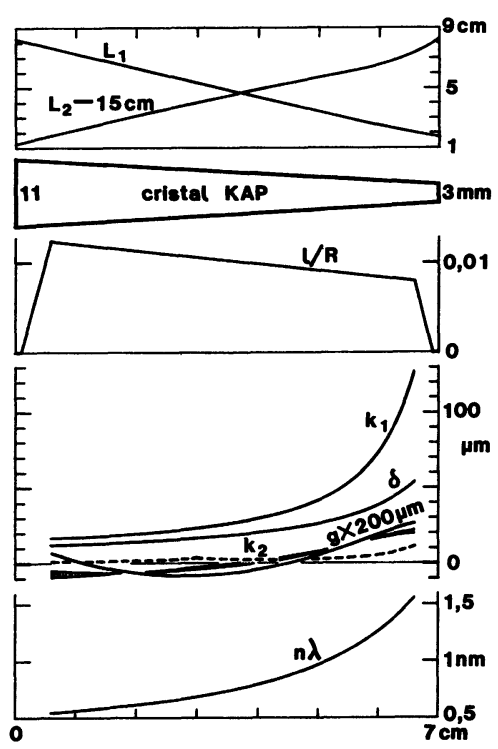

Fig. 2. - Plusieurs paramètres portés en fonction de l'abscisse du point de réflexion sur un cristal trapézoïdal élastiquement fléchi (voir paragraphes 3 et 4).

[Plot of several parameters versus the abscissa of the reflection point on an elastically bent, trapezoidal crystal (see Sects. 3 and 4).]
$1 / R$ (une description plus précise de la surface du cristal, fournie numériquement par la théorie des plaques, serait peu justifiée, car la répartition des contacts aux extrémités est en pratique mal connue).

Nous appliquons ce principe à un cristal trapézoïdal isocèle (Fig. 2), dont la longueur (hauteur du trapèze) est de $70 \mathrm{~mm}$, les bases valant 11 et $3 \mathrm{~mm}$. Pour dégager au mieux le trajet des photons, les appuis de la face concave sont biseautés (Fig. 1) à environ l'angle de Bragg correspondant. Ils occupent respectivement 6 et $4 \mathrm{~mm}$, laissant $60 \mathrm{~mm}$ de longueur optiquement et mécaniquement libre, où la linéarité de $\ell / R$ est représentée sur la figure 2 . Le détecteur est plan et la donnée des courbes $L_{1}$ et $L_{2}$ achève de fixer la configuration, qui assure avantageusement une incidence presque normale sur le détecteur $\left(|\beta|<10^{\circ}\right.$ partout) et la quasi constance du grandissement transversal

$$
G=\left(L_{1}+L_{2}\right) \cos \alpha / L_{0}-1,
$$

où $L_{0}$ est la distance de la source à la fente collimatrice assurant la résolution spatiale, et $\alpha$ l'angle d'incidence du rayon considéré sur la fente (ici, $L_{0}=9,6 \mathrm{~mm}$ donne $G=24$ ). On présente également la courbe de longueur d'onde pour le cristal de KAP ( $2 d=2,66 \mathrm{~nm})$ utilisé ici.

Pour porter $g D$ en trait plein sur la figure 2 , on a choisi le diamètre $D=200 \mu \mathrm{m}$, qui est le maximum pour les microballons étudiés. Le pouvoir de résolution intrinsèque du cristal donne une largeur $\Delta x=k_{1}$ au premier ordre de diffraction, et $k_{2}$ (en tireté) au deuxième ordre, d'après [3] ; $k_{1}$ augmenterait légèrement (moins de $10 \%$ ) avec les valeurs de [4]. L'élargissement propre au plasma-source comporte au moins l'effet Doppler, donnant $\Delta x=\delta$. L'émetteur représentatif choisi est le silicium, avec une température ionique de $500 \mathrm{eV}$, correspondant à un pouvoir de résolution $\lambda / \Delta \lambda=3070$ (indépendant de $\lambda$, donc $\delta$ ne dépend pas explicitement de $n$ ).

On voit qu'au premier ordre de diffraction, le principal effet limitatif est le pouvoir de résolution propre du cristal. Au deuxième ordre (ainsi qu'aux ordre supérieurs), c'est l'élargissement Doppler des raies de la source. L'élargissement géométrique atteint au plus la moitié de ce dernier. Enfin, le pouvoir séparateur du détecteur (fréquemment 20 à $50 \mu \mathrm{m})$ joue plus ou moins selon la position sur le spectre.

\section{Influence de la pesanteur.}

Sous l'effet de son propre poids, le cristal se déforme légèrement par rapport au calcul précédent. On montre aisément qu'un trapèze encastré sur ses bases acquiert une courbure supplémentaire :

$$
\frac{\rho \gamma s_{u u}}{h^{2}}\left\{L \frac{2 u-L}{3}\left[\frac{6}{\varepsilon}-\frac{\varepsilon^{2} L(L+\varepsilon u)^{-1}}{(2+\varepsilon) \ln (1+\varepsilon)-2 \varepsilon}\right]+2 u^{2}-L^{2}\right\}
$$


où $\rho$ est la masse volumique, $h$ l'épaisseur (ici $0,3 \mathrm{~mm}), \gamma$ la composante de l'accélération gravitationnelle normale à la surface, $1+\varepsilon$ le rapport $(>1)$ des bases, $u$ l'abscisse à partir de la petite base vers la grande. Pour une fixation réaliste à quatre appuis simples (supprimant toute compression parasite, génératrice de casse !), une excellente approximation est de prendre comme longueur $L$ la distance entre appuis intérieurs augmentée à chaque bout du tiers de l'intervalle restant jusqu'à l'appui extérieur.

Enfin, $s_{u u}$ est la susceptibilité élastique $s_{11}$ relative à l'axe $u$ (et serait l'inverse du module d'Young pour un matériau isotrope). Ignorant l'orientation cristallographique du KAP dans son plan de clivage, on peut seulement dire, d'après les formules de [5] et les données de [6], que $s_{u u}$ peut aller de 72 à $104 \mathrm{TPa}^{-1}$. Le cristal étant ici penché de $21^{\circ}$, $\rho \gamma s_{u u} / h^{2}$ va donc de 12 à $17 \mathrm{~m}^{-3}$. La bande hachurée de la figure 2 représente les valeurs de $g D$ ainsi corrigées. On voit que le cas présent, où la concavité est tournée vers le bas, est plutôt favorable. Par contre, si l'on retournait le spectrographe, la pesanteur dégraderait légèrement la situation. Il est clair également qu'un environnement vibratoire serait à déconseiller.

Notons encore à titre d'exemple que pour un cristal de $\mathrm{Si}(111)$ dans les mêmes conditions, $\rho \gamma s_{u u} / h^{2}$ ne vaudrait que $1,4 \mathrm{~m}^{-3}$. La gravité ayant donc un effet presque imperceptible, et en faisant abstraction des contraintes de champ optique minimal et d'encombrement, on pourrait pousser l'optimisation du trapèze à son terme : les bases seraient dans le rapport 9 , la courbe $g$ aurait 4 extrema au lieu de 3 , et le maximum de $|g| / \delta$ serait diminué d'un facteur 2 . On pourrait même s'amuser à chercher une forme à bords ondulés annulant $g$ partout, mais il serait sans doute illusoire de vouloir la réaliser, car, sans parler de la précision requise au montage, une simple variation locale de $1 \%$ sur l'épaisseur (dont c'est le cube qui joue sur $R$ ) ferait varier $g$ de plus de 0,03 . Pour la même raison, il serait malsain de vouloir gouverner la courbure par des variations d'épaisseur plutôt que de largeur, surtout pour un cristal clivé où la constance de l'épaisseur s'impose d'elle-même.

\section{Réalisation mécanique.}

Le spectrographe que nous avons construit selon les principes précédents a reçu le nom de PABUTEC (petit analyseur de Bragg ultra-résolvant à trapèze élastiquement courbé). Son emploi dans l'enceinte expérimentale de compression de microballons par six faisceaux laser imposait quelques contraintes, notamment l'exiguïté de l'espace disponible entre les faisceaux et les porte-lentilles. Or la forme trapézoïdale du cristal répond élégamment à cette contrainte, car il se trouve fort heureusement que le trapèze a la bonne orientation, avec la petite base proche de la source!

Par ailleurs, la remise à l'air de l'enceinte tendant à dérégler les repères de focalisation, nous avons décidé de réaliser les chargements et déchargements du film photographique sans rupture du vide principal, grâce à un sas. Mais comme le détecteur est traversé perpendiculairement par un rayon de la sphère d'enceinte, une simple translation radiale $\mathrm{du}$ film $\left(160 \times 35 \mathrm{~mm}^{2}\right)$ aurait nécessité une vanne de diamètre prohibitif. C'est pourquoi la cassette photographique, après son introduction et le pompage du sas, est transférée en longueur suivant un rayon de la sphère, puis bascule d'un angle droit à l'aide d'un système de cames et bielles; en fin de course, la rencontre d'un ergot ouvre le volet d'étanchéité optique.

La photographie (Fig. 3) montre l'ensemble du dispositif, et le schéma éclaté met plus spécialement en évidence la phase de basculement du châssis photographique. A gauche, le boîtier effilé contenant le cristal est relié au cadre récepteur du portefilm par des réglages fins et par un soufflet opaque en caoutchouc. Puis des cornières ajourées rejoignent le flasque circulaire servant à fixer l'ensemble sur l'enceinte. En continuant vers la droite, on trouve la vanne électro-pneumatique $(\varnothing 63 \mathrm{~mm})$, le sas, un soufflet métallique encadré de trois vis pour le réglage grossier, et enfin le manchon magnétique puissant (50 daN) qui entraîne de l'extérieur la canne de transfert.

\section{Résultats expérimentaux.}

Le spectre montré en illustration (Fig. 4) est obtenu avec un microballon de verre (diamètre $200 \mu \mathrm{m}$, épaisseur $1,5 \mu \mathrm{m}$ ) contenant du néon sous 10 bars,

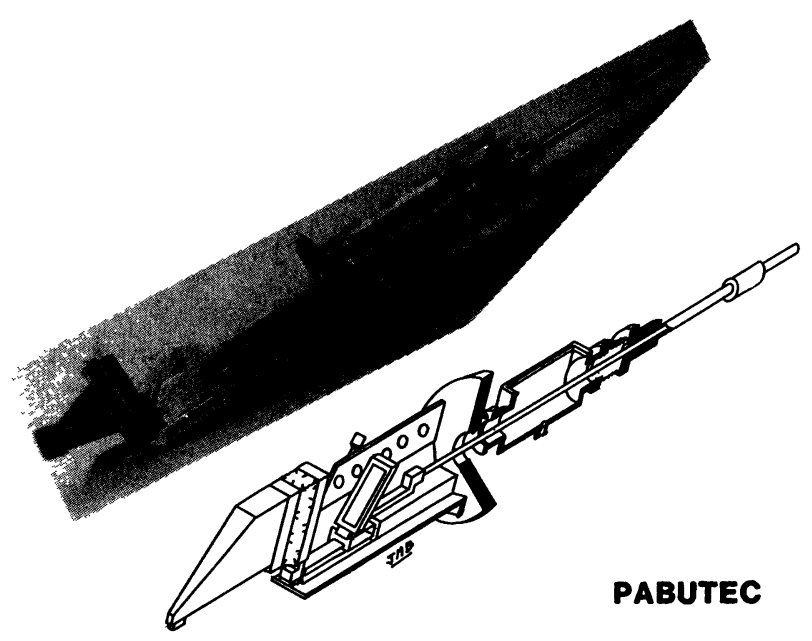

Fig. 3. - Le spectrographe et le système de transfert du porte-film.

[The spectrograph and the transfer device for the filmholder.] 


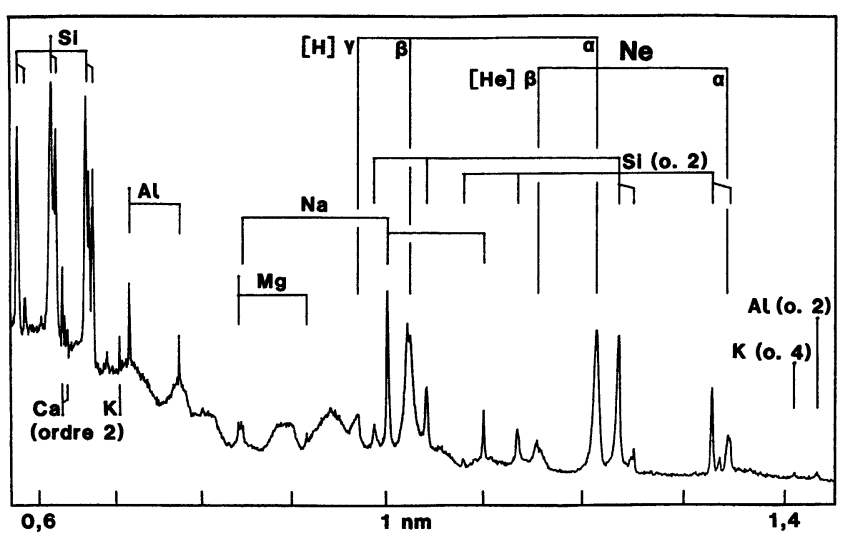

Fig. 4. - Spectre d'implosion par laser d'un microballon de verre contenant du néon.

[Spectrum from a laser-imploded, neon-filled glass microballoon.]

implosé par six faisceaux laser ultra-violets (énergie totale $200 \mathrm{~J}$, en $500 \mathrm{ps,} \mathrm{à} 263 \mathrm{~nm}$ ). Réservant une discussion détaillée à une publication ultérieure, nous ne ferons ici que quelques commentaires.

On est immédiatement frappé par le fort élargissement Stark des raies du néon, d'où l'on déduit une densité électronique d'environ $4 \times 10^{22} \mathrm{~cm}^{-3}$, correspondant à plus d'un facteur 10 de compression volumique, alors que la focalisation était loin d'être optimisée.

Le spectre continu pour $\lambda<0,95 \mathrm{~nm}$ (recombinaison du néon nu, plus deuxième ordre du silicium), qui donne un noircissement croissant vers les courtes longueurs d'onde (transmission de la fenêtre en béryllium de $10 \mu \mathrm{m}$, réponse photographique, etc...), est malheureusement entaché d'ondulations anormales dues à des obstructions partielles de la fente $(20 \mu \mathrm{m})$, peut-être par les débris de supports de cibles issus de la vingtaine de tirs effectués avant le tir présent. D'ailleurs, lors d'une campagne précédente, la fenêtre en béryllium, placée avant la fente et à $9 \mathrm{~mm}$ de la cible, avait été percée en deux ou trois tirs, alors qu'elle est maintenant bien protégée, la fente ne l'étant plus...

On note enfin la présence de nombreux éléments contenus dans le verre du microballon, certains dans plusieurs ordres de diffraction. En particulier, on peut relier l'apparition de la raie de résonance héliumoïde du potassium à l'ordre 4 au pic de réflectivité, étroit et fort $[3,7]$, centré sur le flan d'absorption $K$ du même potassium contenu dans le cristal de KAP.

\section{Conclusion.}

Dans un spectromètre à diffraction de Bragg pour rayons $\mathrm{X}$, la flexion élastique du cristal découpé en trapèze est une méthode simple pour se débarrasser des pertes de résolution spectrale dues aux dimensions de la source, que celle-ci soit un plasma produit par laser ou toute autre source de taille submillimétrique. En outre, la planéité du champ est un avantage décisif pour bon nombre de détecteurs, ainsi que l'incidence quasi normale, sans laquelle la pénétration de rayons $X$ assez durs dans l'épaisseur du détecteur (film avec une couche d'émulsion sur chaque face, par exemple) dégraderait aussi la résolution.

\section{Bibliographie}

[1] Poquérusse, A., Opt. Commun. 58 (1986) 108.

[2] Lemonnier, M., Fourme, R., Rousseaux, F. et KAHN, R., Nucl. Instr. Meth. 152 (1978) 173.

[3] Burek, A. J., Space Sci. Instr. 2 (1976) 53 ; errata 3 (1977) 109.

[4] Henke, B. L., Yamada, H. T. et Tanaka, T. J., Rev. Sci. Instr. 54 (1983) 1311.
[5] Hearmon, R. F. S., An Introduction to Applied Anisotropic Elasticity, (Oxford University Press) 1961.

[6] LANDOLT-BÖRnSTEIN, New Series, group III, vol. 11, « Elastic ... constants ... of crystals », (SpringerVerlag, Berlin, Heidelberg, New York), 1979.

[7] Lewis, M., MaKsym, P. A. et Evans, K. D., Astron. Astrophys. 87 (1980) 213 ; erratum 97 (1981) 218. 